\title{
CATEGORICAL CONSTRUCTIONS IN GRAPH THEORY
}

\author{
RICHARD T. BUMBY \\ Department of Mathematics \\ Rutgers University \\ New Brunswick, New Jersey 08903 \\ and \\ DANA MAY LATCH \\ Department of Mathematics \\ North Carolina State University \\ Raleigh, North Carolina 27650 \\ (Received January 9, 1984)
}

ABSTRACT. This paper presents some graph-theoretic questions from the viewroint of the portion of category theory which has become common knowledge. In parcicular, the reader is encouraged to consider whether there is only one natural category of graphs and how theories of directed graphs and undirected graphs are related.

KEY WORDS AND PHRASES. Category of graphs, algebraic structure. 1980 MATHEMATICS SUBJECT CLASSIFICATION CODES. 05C99, 18B99, $18 C 99$.

\section{INTRODUCTION}

The use of category theory in graph theory is no longer novel (P. Hell [1]), and methods of category theory have been made generally avallable to the "Working Mathematician". The main category theoretic topics that we need and the section number of MacLane [2] in which they may be found are: adjoint functors (Chap. IV); representable functors (Sect. III.2); Kan extensions (Chap. IX); functor categories (Sect. II.4); monads (Chap. VI); and cartestan closed categorles (Sect. IV.6). Nevertheless, one can find examples in the literature of errors, imprecision, or laborious constructions of special cases. As a result of reviewing some of this work for Mathematical Reviews, we set ourselves the task of presenting a sound description of some of the material which seemed most susceptible to these flaws.

In particular, we wish to answer the question in the title of the paper of Harary and Read [3] with a firm "No:", and elaborate on defects noted in the reviews of Farzan and Waller [4] and Ribenboim [5] (see also Ribenbolm [6]). The concepts of "involutorial graph" and "hom-graph" are defined by Ribenboim without citing any earlier work. This makes it difficult to assess their pedigree. We shall present evidence that, as originally presented, these definitions were wrong since they did not account for the category theoretical properties which have been recognized as essential parts of the algebraic structure and cartesian closedness. The difficulty with a study of a "category of graphs" is that graph theory was born as a branch of 
combinatorics which leans towards defining graphs in terms of adjacency properties of a set of vertices, which restrict the avallable objects and ignores the relations between them, while category theory emphasizes the structure preserving mappings (morphisms) and gives lts best results where one is able to construct objects "freely determined by some properties".

Our first observation is that directed graphs are easier to describe than undirected graphs. In Section 2, we construct categories which give satisfactory descriptions of a category of directed graphs and which have simple categorical descriptions (as functor categorfes). The construction of a category of undirected graphs is discussed in Section 3.

Second, vertices and edges have traditionally been considered to be different things, but Ribenboim treated vertices as being degenerate edges. In Section 2 , we study the relationship between the categories of directed graphs which model these two definitions. In Section 4, we observe that the "Cartesian" structure has more intuitively satisfying properties when the vertices are considered as degenerate edges.

Section 3 is devoted to recapturing an undrected graph from one of its canonically generated directed graphs. This process uses the theory of monads which stems from the definition of "algebraic structure" In the context of category theory. 2. TWO POSSIBLE DEFINITIONS OF "DIRECTED GRAPH"

In order to describe a directed graph $G$, one first specifles a set $V$ of vertices and a set $E$ of edges. Each edge is considered as starting at a vertex, called its orlgin and going to another vertex, called its terminal. Actually, these assignments define functions $o$ (for origin) and $t$ (for terminal) from $E$ to $V$. This definition allows oriented graphs to have multiple edges $\left(e_{1}, e_{2} \in E\right.$ with $e_{1} \neq e_{2}, o\left(e_{1}\right)=o\left(e_{2}\right)$ and $\left.t\left(e_{1}\right)=t\left(e_{2}\right)\right)$ and loops $(e \in E$ with $o(e)=t(e))$. often, such graphs are excluded in comblnatorial graph theory problems.

This definition is equivalent to defining a directed graph as a functor from the category $\stackrel{\mathrm{A}}{=}^{\mathrm{OP}}$ (see Figure 1) to the category Ens, of sets.

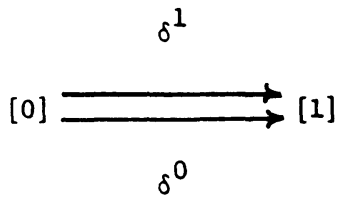

Figure 1. The category $\stackrel{A}{=}$

Clearly, $G[0]$ corresponds to $V$ and $G[1]$ to $E$. Then, without loss of generality, the functions $G\left(\delta^{1}\right): G[1] \rightarrow G[0]$ and $G\left(\delta^{0}\right): G[1] \rightarrow G[0]$ are the maps origin and terminal, respectively.

If graphs are functors, then the approprlate definition of "graph morphism" should be a natural transformation between these functors. Such a natural transformation

$$
\mathrm{f}: \mathrm{G}_{1} \dot{\rightarrow} \mathrm{G}_{2}: \mathrm{A}^{\mathrm{op}} \rightarrow \mathrm{Ens}
$$

is given by a map of vertices $f[0]: G_{1}[0] \rightarrow G_{2}[0]$ and a map of edges $f[1]: G_{1}[1] \rightarrow G_{2}[1]$ which "commute with" origin and terminal. Thus a category of directed graphs can be modeled by the functor category $\left[\underline{A}^{\text {OP }}\right.$, Ens]. 
By contrast, considering vertices to be "degenerate edges" leads one to view the category of graphs as the functor category $\left[\underline{B}^{\text {OP }}\right.$, Ens $]$ (see Figure 2).

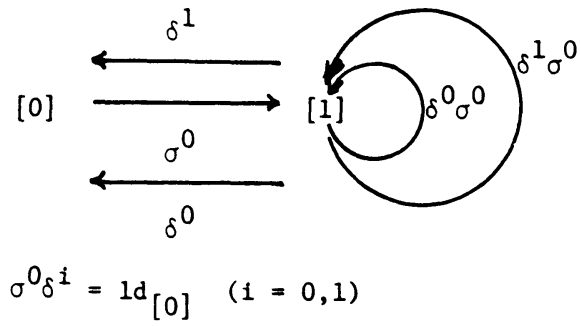

Figure 2. The Category $\stackrel{B}{=}$

If $\mathrm{H}: \underline{\mathrm{B}}^{\mathrm{OP}} \rightarrow$ Ens, then $\mathrm{H}[0]$ represents the vertices of $\mathrm{H}$ and $\mathrm{H}[1]$, the edges of $H$. The functions $H\left(\delta^{1}\right): H[1] \rightarrow H[0]$ and $H\left(\delta^{0}\right): H[1] \rightarrow H[0]$ are again said to be origin and terminal, respectively. In addition, the identities $\sigma^{0} \delta^{1}=1 \mathrm{~d}[0], 1=0,1$ in $\underline{B}$ insure that the function $H\left(\sigma^{0}\right): H[0] \rightarrow H[1]$ is an embedding of the set of vertices into the set of edges of $H$, mapping each vertex to a loop based at that vertex. Thus, each vertex can be thought of as a "degenerate edge".

Since $\left[\underline{\underline{A}}^{O P}, E n s\right]$ and $\left[\underline{B}^{O P}, E n s\right]$ are both examples of functor categorles, we are able to use general propertles of functor categorles to describe the fundamental category-theoretic constructions within each category, and to relate these two candidates for a category of directed graphs. The first principle which we use is that limits and colimits (including the terminal object as a special case of a limit and the initial object as a special case of a colimit) are computed "pointwise".

For example, the null set $\emptyset$ is an inftial object in Ens since there is a unique (null) map of $\emptyset$ into every set. An initial object in [ $\underline{A}^{O P}$, Ens] or $\left[\underline{B}^{\circ P}\right.$, Ens] is a graph with no vertices, no edges, and all structural maps null. The null graph is thus essential to a categorical approach.

Similarly, since a terminal object of $E n s$ is any one pointed set, the terminal object of $\left[\stackrel{A}{=}^{O P}\right.$, Ens] must have one vertex and one edge which is a loop. In $\left[\underline{B}^{O P}\right.$, Ens], the unique edge of the terminal object $T$ is degenerate. Thus $T$ has the property of representing the vertex set of a graph, 1.e., H[0] and the hom-set $\left[\underline{B}^{O P}, E n s\right](T, H)$ are isomorphic as sets, for each graph $H$. In fact, $[0]:\left[\underline{B}^{O P}, E n s\right] \rightarrow$ Ens and $\left[\underline{B}^{O P}, E n s\right](T, \ldots):\left[\underline{\underline{B}}^{O P}, E n s\right] \rightarrow$ Ens are functors which are naturally equivalent.

Since a product in a category is a limit, products in $\left[\underline{A}^{O P}\right.$, Ens] and $\left[\underline{\underline{B}}^{O P}\right.$, Ens] are also computed "pointwise". The product $P=G \times G^{\prime}$ of $A-g r a p h s ~ G$ and $G^{\prime}$ has vertices $P[0]=G[0] \times G^{\prime}[0]$ and edges $P[1]=G[1] \times G^{\prime}[1]$. The origin and terminal maps from $P[1]$ to $P[0]$ are constructed canonically from these maps in $G$ and $G$ '

EXAMPLE. Suppose $G$ and $G^{\prime}$ each have one edge connecting two distinct vertices (e from $a$ to $b$ and $e^{\prime}$ from $a^{\prime}$ to $b^{\prime}$, respectively). Then $P[0]=\left\{\left(a, a^{\prime}\right),\left(a, b^{\prime}\right),\left(b, a^{\prime}\right),\left(b, b^{\prime}\right)\right\} ; P[1]=\left\{\left(e, e^{\prime}\right)\right\} ; P\left(\delta^{1}\right)\left(e, e^{\prime}\right)=\left(a, a^{\prime}\right) ;$ and $P\left(\delta^{0}\right)\left(e, e^{\prime}\right)=\left(b, b^{\prime}\right)$ (see Figure 3$)$. 


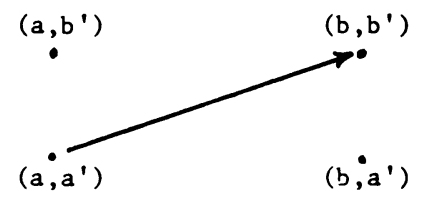

Figure 3. The A-graph $Q \times G^{\prime}$

EXAMPLE. The product $Q$ of $\underline{B}-g r a p h s ~ H$ and $H^{\prime}$ has $Q[0]=H[0] \times H^{\prime}[0]$ and $Q[1]=H[1] \times H^{\prime}[1]$ with $Q\left(\delta^{0}\right), Q\left(\delta^{1}\right)$ and $Q\left(\sigma^{0}\right)$ constructed from the corresponding maps in $H$ and $H^{\prime}$. In particular, if $H$ and $H^{\prime}$ each have two vertices and one nondegenerate edge, then the product graph $Q$ has four vertices and nine edges, four of which are degenerate (see Figure 4).

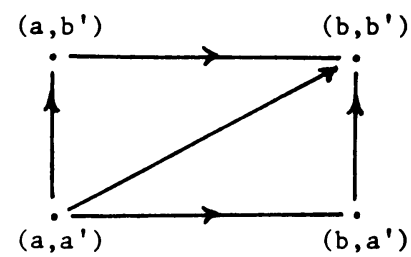

Figure 4. The B-graph $\mathrm{H} \times \mathrm{H}^{\prime}$

Since the same construction can product very different results in $\left[\stackrel{A}{A}^{O P}\right.$, Ens] and $\left[\underline{B}^{\circ p}\right.$, Ens], it is desirable to be able to relate these two categories so that constructions in one would be naturally "induced" in the other.

The inclusion $u: \stackrel{A}{=} \underset{B}{\underline{B}}$ of $\stackrel{A}{=}$ as a subcategory of $\underline{B}$ induces a (forgetful) functor

$$
U:\left[\underline{B}^{O P}, E n s\right] \rightarrow\left[\underline{A}^{O P}, E n s\right] .
$$

For a B-graph $\mathrm{H}$, the $\stackrel{A}{=}-g r a p h ~ U(H)$ is just the restriction of $H$ to the subcategory $\stackrel{A}{=}^{O P}$ of $\stackrel{B}{O}^{O P}$. In the $A$-graph $U(H)$, the degenerate edges $H\left(\sigma^{0}\right) v$, for various vertices $\mathrm{v} \in \mathrm{H}[0]$, are no longer distinguished. The restriction of natural transformations gives no difficulty, and it is easy to verify that $\mathrm{U}:\left[\underline{\mathrm{B}}^{\mathrm{OP}}, E_{\mathrm{n} s}\right] \rightarrow\left[\stackrel{\mathrm{A}}{\mathrm{OP}}^{\mathrm{O}}\right.$, Ens $]$ is actually a functor.

Because $U:\left[\underline{B}^{O P}, E n s\right] \rightarrow\left[\underline{A}^{O P}, E n s\right]$ is defined by composition with the inclusion $\mathrm{u}^{\mathrm{OP}}: \mathrm{A}^{\mathrm{OP}} \rightarrow \underline{\underline{B}}^{\mathrm{OP}}$, it induces more structure: $U$ has both left and right adjoints which are the Kan extensions $L$ and $R$. These are described below.

For each $\stackrel{A}{=}$-graph $G, L(G)$, the "B-graph freely generated by $G$ ", is obtained by adding to $G[1]$ a new degenerate edge $\hat{v}$, one for each vertex $v \in G[0]$, and

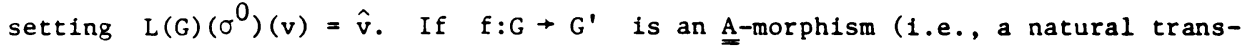
formation from the functor $G$ to the functor $\left.G^{\prime}\right)$, then $L(f): L(G) \rightarrow L_{\left(G^{\prime}\right)}$ is defined to agree with $f$ when "restricted to $G$ " and to satisfy

$$
(L(f)[1])(\hat{v})=(\widehat{f[0])(v)} \text {. }
$$

To see that $L:\left[\underline{A}^{O P}, E n s\right] \rightarrow\left[\underline{B}^{O P}, E n s\right]$ is the left adjoint of $U:\left[\underline{B}^{O P}, E n s\right] \rightarrow\left[\underline{A}^{O P}, E n s\right]$, one shows that there is a natural one-to-one correspondence 


$$
\psi(G, H):\left[\underline{B}^{O P}, E n s\right](L(G), H) \rightarrow\left[\underline{\underline{A}}^{\text {OP }}, E n s\right](G, U(H))
$$

for each $\stackrel{A}{=}$-graph $G$ and each $\stackrel{B}{=}$-graph $\mathrm{H}$.

This adjoint relationship may also be described in terms of the unit natural transformation

$$
\eta: 1 \mathrm{~d} \rightarrow \mathrm{UL}:\left[\underline{\underline{A}}^{\mathrm{op}}, E_{\mathrm{ns}}\right] \rightarrow\left[\underline{\underline{A}}^{\mathrm{op}}, E_{\mathrm{ns}}\right]
$$

or the counit natural transformation

$$
\varepsilon: L U \stackrel{\rightarrow}{\rightarrow} 1 d:\left[\underline{\underline{B}}^{\text {op }}, \text { Ens }\right] \rightarrow\left[\underline{\underline{B}}^{\text {op }}, E n s\right]
$$

of the adjoint pair $<L, U>$. We now construct both natural transformations. The

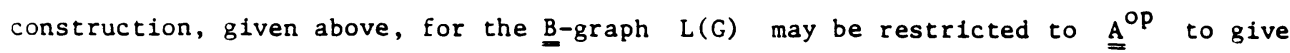
the A-graph UL(G). This construction yields $G$ as a specific subgraph of UL(G); the collection of these inclusions $\eta_{G}: G \rightarrow U L(G)$ is natural in $G$. In fact, this collection is the unit natural transformation of the adjunction $\langle L, U\rangle$. Similarly, the counit $\varepsilon: L U \dot{\rightarrow}$ ld is given by describing the $\underline{\underline{B}-g r a p h}$ morphism $\varepsilon_{H}: L U(H) \rightarrow H$ for each $\underline{B}$-graph $H$. The graph $L U(H)$ differs from $H$ by the addition of new degenerate edges for each vertex, the old ones having lost their distinction in $U(H)$. The B-graph epimorphism $\varepsilon_{H}$ is the identity on $H \subseteq L U(H)$ and maps each new degenerate $\hat{v}$ added by $L$ to the original degenerate edge $H\left(\sigma^{0}\right)(v)$ in $H[1]$. Next, the adjoint relationship $\langle L, U\rangle$ may be verified by demonstrating that the following composites are identity natural transformations:

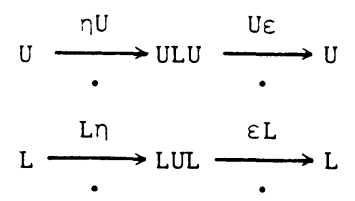

Lastly, note that neither $\varepsilon_{H}$ nor ${ }^{\eta_{G}}$ can be an isomorphism unless $H$ or $G$, respectively, is a null graph.

The right adjoint

$$
R:\left[\underline{\underline{A}}^{\mathrm{OP}}, E_{\mathrm{ns}}\right] \rightarrow\left[\underline{\underline{B}}^{\mathrm{OP}}, E_{\mathrm{ns}}\right]
$$

is given as follows: for each $\stackrel{A}{=}-g r a p h ~ G, R(G)$ is the B-graph whose vertex set $R(G)[0]$ is the collection of all loops of $G$ (i.e. edges $\ell \in G[1]$ with $\left.G\left(\delta^{1}\right)(\ell)=G\left(\delta^{0}\right)(\ell)\right)$. The set of edges $R(G)[1]$ is the collection of all triples $\left\langle\ell_{0}, e, l_{1}\right\rangle$ of edges of $G$ with $\ell_{0}, \ell_{1}$ loops and $e$ an edge having origin the vertex of $\ell_{0}$ and terminal the vertex of $\ell_{1}$, i.e. $G\left(\delta^{1}\right)\left(\ell_{0}\right)=G\left(\delta^{0}\right)\left(l_{0}\right)=G\left(\delta^{1}\right)(e)$ and $G\left(\delta^{1}\right)\left(l_{1}\right)=G\left(\delta^{0}\right)\left(l_{1}\right)=G\left(\delta^{0}\right)(e)$. The origin and terminal of $\left\langle\ell_{0}, e, l_{1}\right\rangle$ are $\ell_{0}, \ell_{1}$, respectively; i.e. $\left.(R(G))\left(\delta^{1}\right)<\ell_{0}, e, \ell_{1}\right\rangle=\ell_{0}$ and $(R(G))\left(\delta^{0}\right)\left\langle\ell_{0}, e, \ell_{1}>=\ell_{1}\right.$. Lastly, the distinguished $R(G)$-loop at the $R(G)$-vertex $\ell$ is $<\ell, \ell, \ell>$; $1 . e$. $(R(G))\left(\sigma^{0}\right)(\ell)=\langle\ell, \ell, \ell\rangle$. The unit natural transformation $\eta: 1 d^{\circ} \rightarrow R U:\left[\underline{B}^{O P}, E n s\right] \rightarrow\left[\underline{B}^{\circ p}, E n s\right]$ consists of the $\underline{\underline{B}}-$ morphisms $\eta_{H}: H \rightarrow R U(H)$. Particularly, $\eta_{H}$ carries a vertex $v$ of the $\underline{B}-g r a p h \quad H$ to the vertex of $R U(H)$ which is the degenerate loop $H\left(\sigma^{0}\right)(v), 1 . e . \eta_{H}(v)=H\left(\sigma^{0}\right)(v)$; and $\eta_{H}$ maps an edge $e$ of $H$ with origin $v$ and terminal $w$ to the edge $\left\langle H\left(\sigma^{0}\right) v, e, H\left(\sigma^{0}\right) w\right\rangle$, i.e. $\eta_{H}(e)=\left\langle H\left(\sigma^{0} \delta^{1}\right) e, e, H\left(\sigma^{0} \delta^{0}\right) e\right\rangle$. Clearly, $\eta_{H}: H \rightarrow R U(H)$ is the B-monomorphism which identifies $H$ with the full subgraph of $R U(H)$ generated by vertices $\left\{H\left(\sigma^{0}\right) v \mid v \in H[0]\right\}$. 
The counit $\varepsilon: U R{ }^{\circ} \rightarrow 1 \mathrm{~d}:\left[\underline{\underline{A}}^{\mathrm{OP}}, E \mathrm{~ns}\right] \rightarrow\left[\underline{\underline{A}}^{\mathrm{Op}}, E \mathrm{~ns}\right]$ is analogously defined to be a family of $\stackrel{A}{=}$-graph morphisms $\varepsilon_{G}: U R(G) \rightarrow G$. The vertices of UR $(G)$ are the loops of $G$. The A-morphism $\varepsilon_{G}$ maps such a loop to its vertex $G\left(\delta^{0}\right)(\ell)=G\left(\delta^{1}\right)(\ell)$ and carries an edge $\left\langle\ell_{0}, e, l_{1}\right\rangle$ to the edge e; 1.e. $\varepsilon_{G}[0](l)=G\left(\delta^{0}\right)(l)$ and $\varepsilon_{G}[1]<\ell_{0}, e, l_{1}>=e$. Thus $\varepsilon_{G}$ "compresses" UR(G) onto the full $\underline{\underline{A}-s u b g r a p h}$ of $G$ generated by those vertices having loops.

Note that $\varepsilon_{G}$ is an A-graph isomorphism if and only if at each vertex of $G$, there is precisely one loop. Simllarly, $\eta_{H}$ is a B-graph isomorphism exactly when each loop of $H$ is a degenerate loop $H\left(\sigma^{0}\right) v$. Therefore $U$ and $R$ induce an isomorphism between the full subcategories $A$ of A-graphs with exactly one loop per vertex and $B$ of $\underline{\underline{B}-g r a p h s}$ with only degenerate loops.

These ideas above 1llustrate the first part of the following theorem whose second part we use later.

THEOREM 2.1 (Lambek and Rattray). Let $\mathrm{s}: \underline{x} \rightleftarrows \underline{y}: \mathrm{T}$ be a pair of adjoint functors with unit $n: 1 \mathrm{~d}{ }^{\circ} \rightarrow \mathrm{TS}: \underline{X} \rightarrow \underline{X}$ and counit $\varepsilon: S T+1 \mathrm{~d}: \underline{y} \vec{z} \underline{y}$. Then $T$ and $S$ induce an equivalence between $F 1 X(T S, n)=\left\{X \in\right.$ obX $\left.\mid \eta_{X}: X \stackrel{\equiv}{\longrightarrow} T S(X)\right\}$ and $\operatorname{Fix}(\mathrm{ST}, \varepsilon)=\left\{\ddot{Y} \in\right.$ oby $\left.\mid \varepsilon_{\mathrm{Y}}: \mathrm{ST}() \stackrel{\cong}{\longrightarrow} Y\right\}$. Moreover, the following statements are equivalent:

(i) the triple (TS, $n, T \subseteq S$ ) on $\underline{x}$ is idempotent;

(ii) $n \mathrm{~T}$ is a natural isomorphism;

(iii) the cotriple $(S T, \varepsilon, S \cap T)$ on $\underline{y}$ is idempotent;

(iv) $\varepsilon S$ is a natural isomorphism;

(v) TS: $\underline{X} \rightarrow \underline{x}$ factors through the subcategory Fix (TS, $n)$;

(vi) ST: $\underline{y} \rightarrow \underline{y}$ factors through the subcategory $\mathrm{Fix}(\mathrm{ST}, \varepsilon)$.

If these conditions hold, Fix(TS, $n)$ is a reflective subcategory of $\underline{x}$ with reflector the factorization $(v)$ and $\operatorname{Fix}(\mathrm{ST}, \varepsilon)$ is a coreflective subcategory of $\underline{y}$ with coreflector the factorization (vi).

PROOF. See Lambek and Rattray [8], Theorem 1.1.

In the example just discussed, $S: \underline{X} \rightarrow \underline{y}$ is $U:\left[\underline{\underline{B}}^{\mathrm{OP}}\right.$, Ens $] \rightarrow\left[\underline{\underline{A}}^{\mathrm{OP}}\right.$, Ens $]$ and $\mathrm{T}: Y \rightarrow X$ is the right adjoint $\mathrm{R}:\left[\underline{\underline{A}}^{\mathrm{OP}}, \mathrm{Ens}\right] \rightarrow\left[\underline{\underline{B}}^{\mathrm{OP}}\right.$, Ens $]$. The condition for $\operatorname{Fix}(R U, n) \subseteq\left[\underline{\underline{B}}^{\mathrm{OP}}, E n s\right]$ to be reflective and $\mathrm{Fix}(U \mathrm{UR}, \varepsilon) \subseteq\left[\underline{\mathrm{A}}^{\mathrm{OP}}\right.$, Ens $]$ to be coreflective fail. Consider $n_{R G}: R G \rightarrow R U(R G)$ where $G$ is a graph with one vertex $v$ and two loops $\ell_{0}, l_{1}$ at $v$. Then $R G$ has two vertices $\left\{l_{0}, l_{1}\right\}$, each with two loops

$$
\left\{<\ell_{0}, l_{1}, l_{0}>,<\ell_{1}, l_{1}, l_{1}>\right\}, 1=0,1 .
$$

However, $R U(R G)$ has four vertices, the four loops (2.1). Thus $\eta_{R G}$ falls to be an isomorphism and condition (11) above does not hold. In fact, $F 1 x(R U, \eta)$ is not coreflective since the inclusion does not preserve colimits.

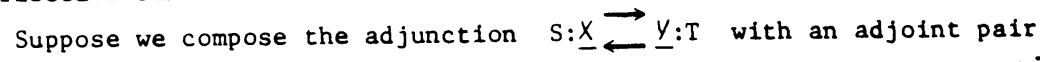
$\mathrm{M}: \underline{y} \rightleftarrows \underline{Z}: \mathrm{N}$ then Theorem 2.1 applies to the composite adjunction $\mathrm{MS}: \mathrm{X} \longrightarrow \mathrm{Z}: \mathrm{NT}$. In particular, if $\underline{z}$ is a reflective subcategory of $\underline{y}$ with idempotent cotriple (MSTN, $\varepsilon, M S \cap T N$ ), then there is a reflective subcategory $\underline{x}_{-1}$ of $\underline{x}$, guaranteed by Theorem 2.1 which is equivalent to a coreflective subcategory $\underline{Z}_{1}$ of $\underline{z}$.

As an illustration, let $\Gamma_{1}$ be the full reflective subcategory of [A ${ }^{\text {OP }}$, Ens] generated by all A-graphs which have at most one loop at each vertex. The left 
adjoint (usually called the reflector) $M:\left[\stackrel{A}{=}^{O P}, E_{n s}\right]+\Gamma_{1}$ of the inclusion $\mathrm{N}: \Gamma_{1} \leftrightarrow\left[\underline{\underline{A}}^{\mathrm{OP}}\right.$, Ens] maps each $\stackrel{\mathrm{A}-g r a p h}{=} G$ to the quotient $\underline{\underline{A}-g r a p h} G_{1}$ with the same vertices as $G$, the same edges between distinct vertices, but having only one loop at each vertex at which $G$ has loops and having no new loops. Applying $U R:\left[\underline{A}^{O P}, E n s\right] \rightarrow\left[\underline{A}^{O P}, E n s\right]$ to a graph $N_{1}$ with $\sigma_{1} \in \Gamma_{1}$, yields the full subgraph $U R\left(G_{1}\right)$ of $N G_{1}$ generated by the vertices of $N G_{1}$ at which there is a loop. Note that $\operatorname{UR}\left(G_{1}\right)$ is already in the subcategory $\Gamma_{1}$; hence the function $M:\left[\underline{A}^{O P}, E n s\right] \rightarrow \Gamma_{1}$, has no further effect and $\operatorname{MURN}\left(G_{1}\right)=\operatorname{URN}\left(G_{1}\right) \hookrightarrow N G_{1}$. Iterating this construction yields the same graph, up to isomorphism. Thus condition (vi) of Theorem 2.1 holds, and all the other equivalent properties $(1)-(v)$ and the conclusions of Theorem 2.1 follow. Particularly, the coreflective subcategory $\Gamma$ of $\Gamma_{1}$ consisting of $A$-graphs with exactly one loop per vertex is equivalent to the reflective subcategory $\Delta$ of B-graphs with only degenerate loops. Note that $\Gamma=F i x(U R, \varepsilon)$ and $\Delta=F i x(R U, \eta)$ for the original adjunction

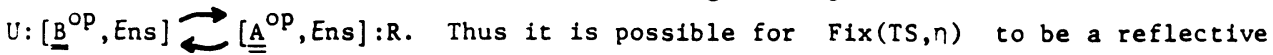
subcategory of $\underline{x}$ without the existence of a factorization of $T S: \underline{X} \rightarrow \underline{x}$ through Fix $(T S, n)$ (i.e. condition (v) of Theorem 2.1 falls even though part of the conclusion is still true).

A second important subcategory $\Gamma_{2}$ of $\left[\stackrel{A}{A P}^{O P}, E n s\right]$ is the full reflective subcategory generated by the simple A-graphs $G$ with at most one edge from any particular vertex to another. The reflector $M:\left[\mathbb{A}^{\mathrm{OP}}\right.$, Ens $]+\Gamma_{2}$, the left adjoint of the full inclusion $\mathrm{N}: \Gamma \underset{2}{\longrightarrow}\left[\stackrel{\mathrm{A}}{\mathrm{OP}}^{\mathrm{P}}, \mathrm{Ens}_{\mathrm{n}}\right]$, maps each $\stackrel{\mathrm{A}-\mathrm{graph}}{=} \mathrm{G}$ to the quotient A-graph $G_{2}$ having the same vertices as $G$, but with edges $e$ and $e^{\prime}$ identified whenever $G\left(\delta^{l}\right) e=G\left(\delta^{l}\right) e^{\prime}$ and $G\left(\delta^{0}\right) e=G\left(\delta^{0}\right) e^{\prime}$. Again, the cotriple on $\Gamma_{2}$ is easily seen to be idempotent. Using an argument similar to the one for $\Gamma_{1}$, it follows that there exists a reflective subcategory $\Delta_{2}$ of $\left[\underline{B}^{\circ p}\right.$, Ens] which is equivalent to a coreflective subcategory $\hat{\Gamma}_{2}$ of $\Gamma_{2}$.

Theorem 2.1 thus establishes equivalences between subcategories of [A ${ }^{\text {op }}$, Ens] and $\left[\underline{B}^{\cup P}\right.$, Ens] which introduce the combinatorially useful concepts of "absence of (unnecessary) loops" or "characterization of edges by their origin and terminal vertices". The "combinatorially interesting graphs" are extracted in a natural way from either of the functor categories $\left[\underline{A}^{O P}, E_{n s}\right]$ or $\left[\underline{B}^{O P}, E n s\right]$. Thus either of the two possible general definitions of "directed graph" leads to the same theory. Along the way, though, we have found that the interplay of these generalizations leads to deeper properties than one might have expected.

3. UNDIRECTED GRAPHS

In combinatorial problems, a graph is simple considered to be one-dimensional complex. Each graph $U$ has a set $V(U)$ of vertices, a set $E(U)$ of edges, and an incidence relation $I(U) \subseteq V(U) \times E(U)$ with each edge incident to at most two vertices. Clearly, a directed A-graph $G$ can be given this structure, denoted by $P(G)$ : the vertices are $G[0]$; the edges are $G[1]$; and the incidence relation is the collection of pairs

$$
\left\{\left(G\left(\delta^{0}\right) \text { e,e }\right),\left(G\left(\delta^{1}\right) \text { e,e }\right) \mid \text { e e } G[1]\right\} \subseteq G[0] \times G[1] .
$$

A morphism $f: U_{1} \rightarrow U_{2}$ of undirected graphs is given by a pair of functions,

$$
V(f): V\left(U_{1}\right) \rightarrow V\left(U_{2}\right) \text { and } E(f): E\left(U_{1}\right) \rightarrow E\left(U_{2}\right) \text {, }
$$


such that incidence is preserved; i.e.

$$
\left(V(f) \times E(f) I\left(U_{1}\right) \subseteq I\left(U_{2}\right)\right.
$$

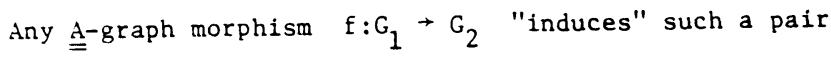

$$
P(f): P\left(G_{1}\right) \rightarrow P\left(G_{2}\right)
$$

of functions between undirected graphs since $f$ commutes with origin and terminal. Hence, this construction induces a functor

$$
P:\left[\stackrel{A}{=}^{O P}, E n S\right] \rightarrow T
$$

from the category of A-graphs to the category $T$ of undirected graphs.

The category $T$ is not easy to describe, since it depends on using, within category theory, the notation of a set with at most two elements (to describe the incidence relation $I(U))$. This difficulty illustrates a difference between the combinatorial and categorical viewpoints in graph theory. The categorical approach searches for general concepts which may be simply described and proceeds from there to particular or special examples; while the combinatorial approach assumes that such things as multiple edges or loops will be complicated. The "purity" of the categorical approach aside, it appears necessary to make some arbitrary choices in order to obtain a category to model $T$. However, we are able to avoid this difficulty by using external relationships (which we expect to exist) between [ $\underline{\underline{A}}^{O P}$, Ens] and $n$ to help "internally" construct $T$.

The functor $P:\left[\underline{A}^{O P}\right.$, Ens $] \rightarrow T$ forgets orientation; hence it is reasonable to investigate the possibility that $P$ has a right adjoint

$$
0: T \rightarrow\left[\underline{\underline{A}}^{\text {op }}, E n s\right] \text {. }
$$

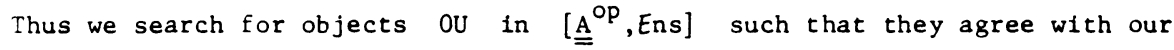
"intuition" about the category $T$ and such that there is a natural equivalence

$$
\psi(G, U): T(P G, U) \stackrel{\simeq}{=}\left[\underline{A}^{\text {OP }}, E n s\right](G, O U) \text {. }
$$

Every A-graph $G$ is determined by the sets $G[0]$ and $G[1]$ of vertices and edges, respectively, and by the functions $G\left(\delta^{1}\right)$ and $G\left(\delta^{0}\right)$ of origin and terminal. Since $\left[\underline{A}^{O P}, E n s\right]$ is a functor category, the Yoneda Lemma guarantees that these sets and functions can be naturally described in terms of representable functors; in particular,

and

$$
G[1]=\left[\underline{\underline{A}}^{o p}, E n s\right](\underline{\underline{A}}(\ldots,[i]), G), \quad i=0,1
$$

$$
G\left[\delta^{1}\right]=\left[\underline{\underline{A}}^{\text {Op }}, E n s\right]\left(\underline{\underline{A}}\left(,, \delta^{i}\right), G\right), \quad i=0,1
$$

The object (actually functor)

$$
\mathrm{V} \equiv \underline{\underline{A}}\left({ }_{-},[0]\right): \underline{\underline{A}}^{O P} \rightarrow \text { Ens }
$$

which represents vertices has one vertex since $\stackrel{A}{=}([0],[0])$ consists only of the identity, and no edges since $\underline{=}([1],[0])$ is empty. The object

$$
E \equiv A(,[1])::^{\text {OP }} \rightarrow \text { Ens }
$$

which represents edges similarly has two vertices and one edge joining them. The morphisms (natural transformations)

$$
\mathrm{B} \equiv \underline{\underline{A}}\left(,, \delta^{1}\right): \underline{\underline{A}}(\ldots,[0]) \dot{\mathrm{A}}\left({ }_{-},[1]\right)
$$


and

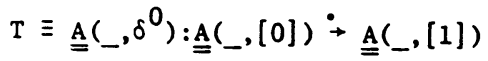

from $V$ to $E$ select the origin and terminal vertices, respectively, of the unique

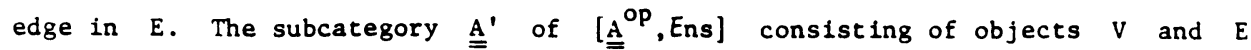
with morphisms $B$ and $I$ is isomorphic to $A$.

For each graph $U$ in $T$, we can determine the structure of $O U$ by using both (3.1) and (3.2). The set of vertices of oU is $\left[\stackrel{\mathrm{A}}{\text { OP }}^{\text {, Ens] }}(\mathrm{V}, \mathrm{OU})\right.$ which is naturally isomorphic to $T(P V, U)$ :

$$
\begin{aligned}
(\mathrm{OU})[0] & \left.\cong\left[\stackrel{\mathrm{A}}{\mathrm{OP}}^{\mathrm{O}}, \text { Ens }\right] \stackrel{(\mathrm{A}}{=}(,,[0]) .0 U\right) \\
& =\left[\underline{\mathrm{A}}^{\text {OP }}, \text { Ens }\right](\mathrm{V}, \mathrm{OU}) \\
& \cong T(P V, U) .
\end{aligned}
$$

Since graph $V$ has one vertex and no edges, and $P:\left[{ }_{A}^{\text {op }}\right.$, Ens $] \rightarrow T$ is a forgetful functor, $P V$ is the graph in $T$ with one vertex and no edges. A $T$-morphism $\mathrm{PV} \rightarrow \mathrm{U}$ is given by selecting a vertex of $U$ to be the image of the unique vertex of PV. Thus (OU)[0] is identified with the set of vertices of $U$.

Similarly, the edge set of $O U$ is given by the set $T(P E, U)$. The $A-m o r p h i s m$ $T: V \rightarrow E$ induces a map $T(P T, U): T(P E, U) \rightarrow T(P V, U)$. The undirected graph $P E$ has one edge and two vertices; thus $T(P T, U)$ restricts a morphism with domain $P E$ to one of these vertices. If vertex $v$ of $U$ is incident to edge $e$, then there is a $T$-morphism

$$
\mathrm{f}_{\mathrm{e}}: \mathrm{PE} \rightarrow \mathrm{U}
$$

such that the unique edge of $P E$ is mapped to $e$ and such that the vertex of $P E$ designated by $\mathrm{PT}: \mathrm{PV} \rightarrow \mathrm{PE}$ is mapped to $\mathrm{v}$.

We expect that this description above would determine the morphism $f_{e}$ uniquely. First, if $e$ is a proper edge, then $e$ has precisely one other vertex incident with it; and $f_{e}: P E \rightarrow U$ should have this vertex as the image of the second vertex of PE. Second, if $e$ is a loop, then there is only one vertex $v$ incident with $e$; and both vertices of $P E$ must be mapped to $v$. Note that these assignments establish a natural equivalence between

$$
(O U)[1] \simeq T(P E, U) \text { and } I(U) \subseteq V(U) \times E(U) \text {. }
$$

In addition, the terminal map

$$
(O U)\left(\delta^{0}\right):(O U)[1] \rightarrow(O U)[0]
$$

is given by the natural projection of $I(U)$ on $V(U)$.

To complete the description of the directed graph $O U$, it is necessary to give the origin map

$$
(O U)\left(\delta^{1}\right):(O U)[1] \rightarrow(O U)[0] \text {. }
$$

The argument given above to justify the identification of $T(P E, U)$ with $I(U)$ is a construction of $(O U)\left(\delta^{1}\right)$; 1 .e.

$$
(O U)\left(\delta^{1}\right)(v, e)=\left\{\begin{array}{l}
v^{\prime} ; \text { if }\left(v^{\prime}, e\right) \in I(U), v^{\prime} \neq v \\
v ; \text { otherwise. }
\end{array}\right.
$$

This construction uses the difficult to naturally describe notion "is incident with either one or two vertices". The existence of the adjoint relationship $\langle P, 0\rangle$ 
demands that the construction of the origin be natural. Hence we will exploit the simple functor category $\left[\underline{\underline{A}}^{\mathrm{OP}}\right.$, Ens] of $\mathrm{A}$-graphs, use the assumed existence of an adjoint pair $\langle P, 0\rangle$, and apply the general theory of monads to construct a category

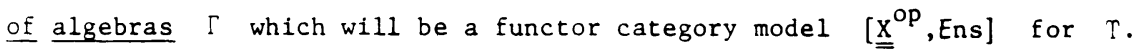

As a first step, we give a description of the monad (or triple) $M=\langle T, n, \mu\rangle$ in $\left[\stackrel{A}{A}^{O P}, E n s\right]$ which results from the assumed existence of the adjunction $\langle P, 0\rangle$. For each $\stackrel{A}{=}$-graph $G, T G \equiv O P(G)$ is described using the above discussion. Hence, $[G[0]=G[0]$ and $T G[1]$ is given as the pushout

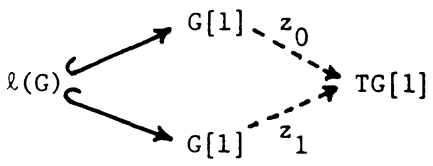

where $2(G)=\left\{e \in G[1] \mid G\left(\delta^{0}\right)(e)=G\left(\delta^{l}\right)(e)\right\}$, the set of loops of $G$. The universal properties of the pushout in $E$ ns are used to give a description of origin $T G\left(\delta^{1}\right)$ and terminal $\mathrm{TG}\left(\delta^{0}\right)$ to reflect the above construction of

$$
\mathrm{T} \equiv \mathrm{OP}:\left[\underline{\underline{\mathrm{A}}}^{\mathrm{OP}}, \mathrm{Ens}\right] \rightarrow\left[\underline{\underline{A}}^{\mathrm{OP}}, \text { Ens }\right] \text {. }
$$

Thus $\mathrm{TG}\left(f^{0}\right): \mathrm{TG}[1] \rightarrow \mathrm{TG}[0]$ is the composition of the identification ${ }_{G}[0]: G[0] \stackrel{\cong}{\rightrightarrows} T G[0]$ with the function $\omega$ in

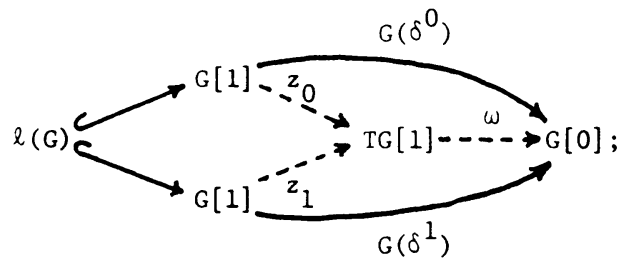

while $T G\left(\delta^{1}\right): T G[1] \rightarrow T G[0]$ is defined similarly by interchanging $G\left(\delta^{0}\right)$ and $G\left(\delta^{1}\right)$ in (3.4).

The unit of the adjunction $\langle P, 0\rangle$,

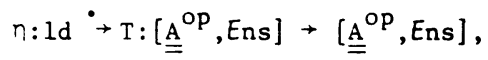

is given by

$$
n_{G}[0] \equiv 1 d: G[0] \rightarrow T G[0] \text {, and } n_{G}[1] \equiv z_{0}: G[1] \rightarrow T G[1]
$$

of diagram (3.3), for each $\stackrel{A}{=}$-graph G. Clearly, from the definition of $T G\left(\delta^{0}\right)$

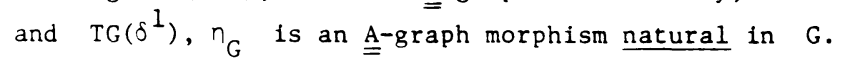

The multiplication

$$
\mu: \mathrm{T}^{2} \rightarrow \mathrm{T}:\left[\underline{\underline{A}}^{\mathrm{OP}}, E \mathrm{~ns}\right] \rightarrow\left[\underline{\underline{A}}^{\mathrm{OP}}, E \mathrm{~ns}\right]
$$

of the monad $M$ is constructed from the counit $\varepsilon: P 0^{\bullet} \rightarrow 1 d: \Upsilon \rightarrow ?$ of the adjoint pair $\langle P, 0\rangle$. For each graph $U, \varepsilon_{U}: P O(U) \rightarrow U$ is the identifying map on vertices, $V\left(\varepsilon_{U}\right) \equiv I d: V(P O(U)) \rightarrow V(U)$. The set $E(P O(U))$ of edges of $P O(U)$ is, from the above discussion, just the incidence relation $I(U) \subseteq V(U) \times E(U)$. A sketch of the construction of the equivalence

$$
\psi(G, U): T(P G, U) \stackrel{\cong}{\longrightarrow}\left[\mathrm{A}^{\mathrm{oP}}, \mathrm{Ens}\right](\mathrm{G}, \mathrm{OU})
$$

leads to the function $E\left(\varepsilon_{U}\right): E(P O(U)) \rightarrow E(U)$ being the projection $I(U) \rightarrow E(U)$. The incidence relation $\mathrm{I}(\mathrm{PO}(\mathrm{U}))$ in $\mathrm{PO}(\mathrm{U})$ can be given as a pushout with a map to $\mathrm{I}(\mathrm{K})$ : 


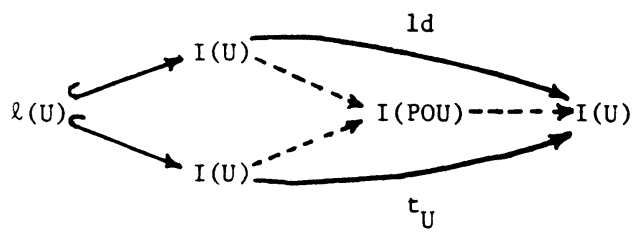

where $t_{U}$ is a "twist" map which chooses the other element of $I(U)$ over the same edge in $E(U)$. In the context of the monad $M$, the above constructions lead to the pushout description (3.6) for the multiplication $n_{G}: T^{2} G \rightarrow T G$ :

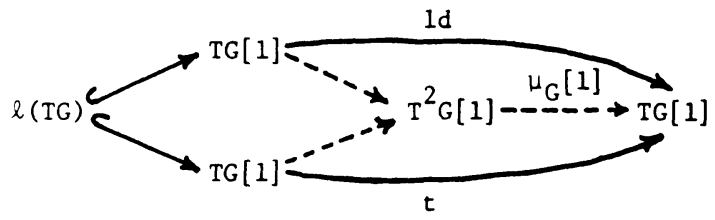

where $t: \operatorname{TG}[1] \rightarrow \operatorname{TG}[1]$ is defined by

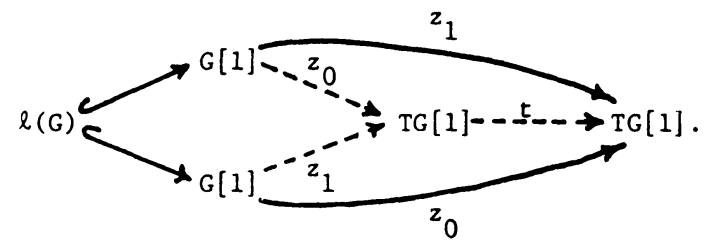

Note that $t: T G[1] \rightarrow T G[1]$ together with $1 d: G[0] \rightarrow G[0] \cong T G[0]$ on vertices does not define an $\stackrel{A}{=}$-graph morphism, but $\mu_{G}=\left\langle 1 d, \mu_{G}[1]\right\rangle: T^{2} G \rightarrow T G$ is in [ $\left.\underline{\underline{A}}^{\text {op }}, E n s\right]$.

The axioms for a monad (see MacLane [2], VI.1) are now easily verified; in particular, the constructions are clearly "natural in $G^{\prime \prime}, G \in\left[A^{\circ p}, E n s\right]$.

An algebra for the monad $M=\langle T, \eta, \mu\rangle$ is defined as an object $X$ of the underlying category, which is $\left[\stackrel{A}{ }^{O P}, E n s\right]$ in this case, together with a morphism $h: T X \rightarrow X$ such that diagrams (3.8) commute:
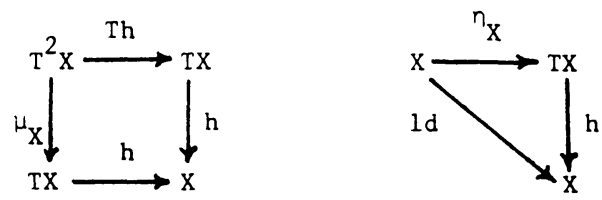

Since $\eta_{X}[0]=1 d: x[0] \rightarrow(T X[0] \equiv X[0])$ and $\eta_{X}[1]: X[1] \rightarrow \operatorname{TX}[1]$ is the map $z_{0}$ of (3.3), we construct $h: T X \rightarrow X$ with $h \eta_{X}=1 d_{X}$ from the pushout (3.9):

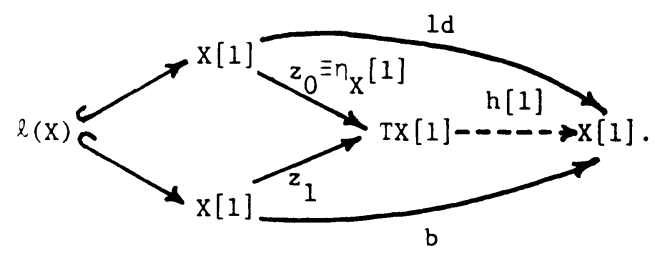

The requirement that $\mathrm{h}: \mathrm{TX} \rightarrow \mathrm{X}$ be an A-graph morphism forces the map $\mathrm{b}: \mathrm{X}[1] \rightarrow \mathrm{X}[1]$ to reverse ortentation (i.e., $\left.X\left(\delta^{1}\right) b=X\left(\delta^{1-1}\right): X[1] \rightarrow X[0], 1=0,1\right)$. Furthermore, the restriction $b / \ell(X)$ must be the "identity" inclusion $\ell(X) \rightarrow X[1]$. The commuting of the first diagram of (3.8) reduces, in this case, to the statement that 
$b: x[1] \rightarrow x[1]$ has

$$
b^{2} \equiv 1 d: x[1] \rightarrow x[1]
$$

i.e., b is an involution.

Thus the concept of graph with involution arises naturally from the concept of unoriented graph; in fact, it provides an algebraic realization of this concept. The category $T_{1}$ of M-algebras always has a pair of adjoint functors which induce the monad $M$ in the base category.

In addition, the Kleisli construction uses the monad $M$ to describe a category ${ }^{I_{2}}$ which is essentlally the category of free algebras. The objects of $T_{2}$ are the same as those of $\left[\stackrel{A}{=}^{O P}\right.$, Ens], but there are additional morphisms in $T_{2}$. Any pair of functions $f=\left\langle f_{0}, f_{1}\right\rangle, f_{0}: G[0] \rightarrow G^{\prime}[0]: f_{1} G[1] \rightarrow G^{\prime}[1]$ which preserve incidence (rather than origin and terminal) is a morphism of $\mathrm{T}_{2}$.

In general, the category of all algebras (in our case, $T_{1}$ ) is a terminal object in the category $\operatorname{Adj}(M)$ of adjoint pairs which induce the monad $M$, and the Kleisli construction (in our case $T_{2}$ ) is initial in $\operatorname{Adj}(M)$. The unique $\psi: T_{2} \rightarrow T_{1}$ functor of MacLane [2] (VI.5, Theorem 3) is both full and faithful. Furthermore, every algebra in $T_{1}$ is isomorphic to a free algebra in $\psi\left(T_{2}\right)$; hence, the two categories are equivalent. Thus, $T_{1}$ and $T_{2}$ are, essentially, equally good models of $T$, and they are canonically chosen from all categories inducing the monad $M$.

The category $T_{2}$ is perhaps closer to our original idea of unoriented graphs $n$; but the category $T_{1}$ has other advantages. Observe that A-graphs with involution

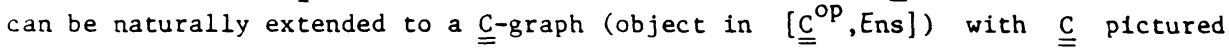
in Fig. 5 .

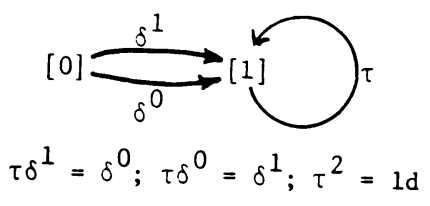

Figure 5. The category $\underline{\underline{C}}$

The category of algebras $T_{1}$ defined by the monad $M$ is the full subcategory of $\left[\underline{C}^{\text {op }}, E n s\right]$ of $\underline{\underline{C}}$-graphs $U$ satisfying $\{e \mid U(\tau) e=e\}=\left\{e \mid U\left(\delta^{0}\right) e=U\left(\delta^{1}\right) e\right\} ; i . e$. , the set of edges fixed by $U(\tau)$ is precisely the set of all loops of $U$. It is easy to show that $T_{1}$ is a reflective subcategory of $\left[\underline{C}^{\text {op }}\right.$, Ens]. Construct the reflector, the left adjoint to the inclusion of $T_{1}$ into $\left[\stackrel{C}{C P}^{\circ}\right.$, Ens], by passing from a given $\underline{\underline{C}-g r a p h} U$ to a new $\underline{\underline{C}}-g r a p h \quad U_{1}$, a quotient of $U$, having the same vertices as $U$, having the same edges between distinct vertices of $U$, but having $U(\tau)$ e Identified with $e$ for each loop e $\in \ell(U)$.

Note that equivalence of categories is weaker than 1somorphism. In particular, the comparison $\psi: T_{2} \rightarrow T_{1}$ is not an isomorphism of categories, 1.e., the object map $|\psi|:\left|T_{2}\right| \rightarrow\left|T_{1}\right|$ is not one-to-one and onto. However, the notion of equivalence appears to be more appropriate than isomorphism for modeling a theory. Hence, there is only one "useful" model up to equivalence, but two different approaches to that model.

We may also form a corresponding theory for $\underline{\underline{B}}-g$ raphs starting from an orientation-forgetful functor

$$
P^{\prime}:\left[\underline{B}^{O P}, E n s\right] \rightarrow T^{\prime} .
$$


The category of algebras $T_{1}^{\prime}$ for a monad $M^{\prime}$ in $\left[\underline{B}^{\circ P}\right.$, Ens], with similar properties to the monad $M$ in $\left[\stackrel{A}{O}^{O P}, E n s\right]$, may be described by introducing an "involution". These algebras approximate the "involutional graphs" of Ribenboim [5]. Of course, if a graph admits more than one involution, each involution defines a different algebra. (The original definition required only the existence of an involution in the hope of defining a subcategory of $\left[\underline{B}^{O P}\right.$, Ens]; but the involution was incorporated into the algebraic structure in Ribenboim [6].) Curiously, the definition in Ribenboim [5,6] also requires that

an edge fixed by involution be degenerate.

The requirement $(3.10)$ is incorporated naturally into the program developing the monad $M^{\prime}$ by having $\mathrm{H}\left(\sigma^{0}\right): \mathrm{H}[0] \hookrightarrow \mathrm{H}[1]$ play the role of the inclusion $\ell(G) \hookrightarrow G[1]$ of (3.3) in the definition of $T^{\prime} H[1]$. With this change, $T^{\prime} H\left(\sigma^{0}\right)$ is the common composition $\mathrm{H}[0] \rightarrow \mathrm{T}^{\prime} \mathrm{H}[1]$ in the analog of (3.3); and $T^{\prime} H\left(\delta^{i}\right): T^{\prime} H[1] \rightarrow T^{\prime} H[0], i=0,1$, is given as in (3.4). Again, $\eta_{H}^{\prime}[1]: H[1] \rightarrow T^{\prime} H[1]$ is the analog of $z_{0}$ of (3.3), and $\mu_{H}^{\prime}[1]: T^{\prime} T^{\prime} H[1] \rightarrow T^{\prime} H[1]$ is constructed as in (3.6) and (3.7). (Note that the mapping $\mu_{H}$, in general, is not as isomorphism (cf. Ribenboim [5], p. 159).)

The category $T^{\prime}$ of all algebras for the monad $M^{\prime}$ in $\underline{B}^{O P}$, Ens ] is the category $\left[\underline{D}^{\mathrm{OP}}, E_{\mathrm{n}}\right]$, where $\stackrel{D}{=}$ is depicted in Figure 6 .

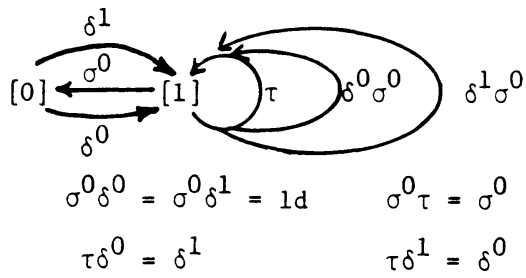

Figure 6. The category $\stackrel{D}{=}$

The corresponding Kleisli construction yields a category $\tau_{2}^{\prime}$ which is not equivalent to $T_{1}^{\prime}$; rather the free algebras in $T_{2}^{\prime}$ are characterized by (3.10). Thus the original definition of involutorial graph was designed to select a subcategory of $T_{1}^{\prime}$ equivalent to $T_{2}^{\prime}$. It is also curious that (3.10) defines a subcategory of $T_{1}^{\prime} \equiv\left[\underline{D}^{\mathrm{OP}}\right.$, Ens $]$ which is both reflective and coreflective.

Also, consider the Kan extensions of the functors induced by the inclusions

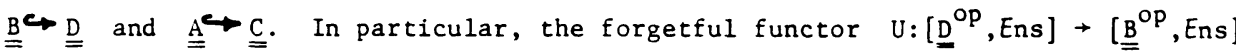
and its left adjoint (i.e., left-Kan extension) induce the monad $M$.

4. CATEGORIES OF GRAPHS ARE CARTESIAN CLOSED

A benefit of viewing a category of graphs as a functor category is that any functor category $\left[\underline{X}^{O P}, E n s\right]$, for small $\underline{X}$ is cartesian closed (see Freyd [9], $\left.p .8\right)$. The proof of this result is constructive; i.e. an algorithm is provided for computing the "internal-Hom" functors. If $Y, Z$ are objects in $\left[\underline{X}^{O P}, E n s\right]$, then $\operatorname{Hom}(Y, Z)$ is also an object in $\left[\underline{x}^{\text {OP }}\right.$, Ens], whose evaluation at object $p$ of $\underline{X}$ is defined by:

$$
\operatorname{Hom}(\mathrm{Y}, \mathrm{Z})(\mathrm{p}) \equiv\left[\underline{\underline{X}}^{\mathrm{OP}}, E_{\mathrm{ns}}\right](\underline{\underline{X}}(\ldots, \mathrm{p}) \times \mathrm{Y}, \mathrm{Z}) .
$$


Thus, for our examples, we give the representable functors

$$
\underline{\underline{X}}(,[1]): \underline{\underline{x}}^{\text {op }} \rightarrow E_{\mathrm{ns}}
$$

for $1=0,1$ and $X=A, B, C$ or $D$. Figure 7 depicts the directed graphs given by these representable functors.

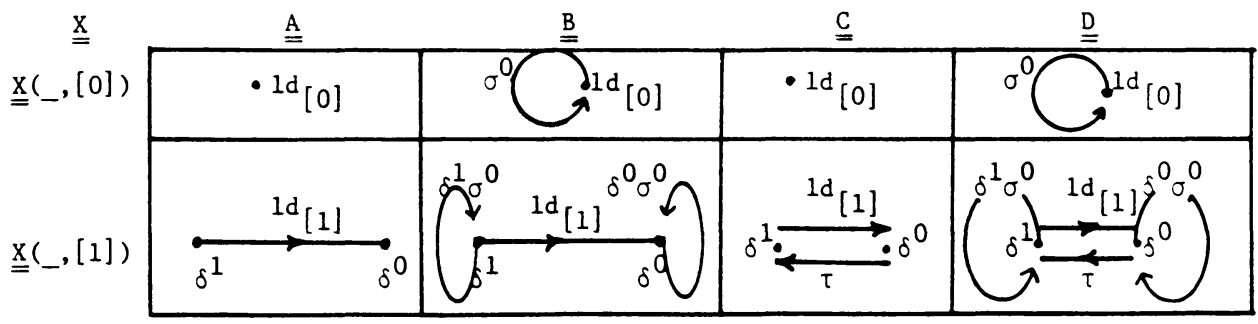

Figure 7. Representable functors

As an example of the use of formula (4.1), we construct $\operatorname{Hom}(Y, Z)$ in $\left[\underline{A}^{O P}, E n s\right]$. The set of vertices is given by

$$
\operatorname{Hom}(Y, Z)[0] \equiv\left[\underline{\underline{A}}^{O P}, E n s\right](A(,[0]) \times Y, Z) .
$$

But $A($, $[0])$ is a graph with one vertex and no edges. Since products are computed "coordinatewise", $A(,[0]) \times Y$ has vertices $Y[0]$ and no edges. Thus

$$
\operatorname{Hom}(Y, Z)[0] \cong \operatorname{Ens}(Y[0], Z[0]) \text {. }
$$

The graph $\stackrel{A}{=}(,,[1])$ has one edge joining two vertices. Thus $\left.\mathrm{A}_{-},[1]\right) \times Y$ has the same number of edges as $Y$, but twice as many vertices. The structural maps satisfy

$$
\delta^{i}(\langle 1 d\rangle, e)=\left(\left\langle\delta^{i}\right\rangle, \delta^{i} e\right) .
$$

Thus $\operatorname{Hom}(Y, Z)[1]$ is identifled with the set of all triples of functions $\langle\mathrm{r}: \mathrm{Y}[1] \rightarrow \mathrm{Z}[1], \mathrm{s}: \mathrm{Y}[0] \rightarrow \mathrm{Z}[0], \mathrm{t}: \mathrm{Y}[0] \rightarrow \mathrm{Z}[0]\rangle$ for which diagrams $(4.2)$ commute

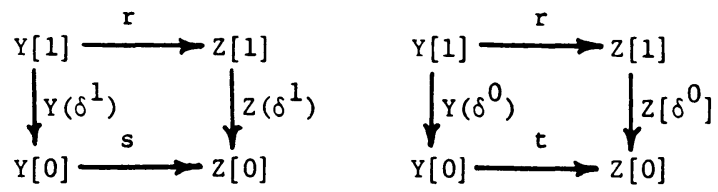

The origin, $\operatorname{Hom}(Y, Z)\left(\delta^{1}\right)$, is given by

$$
<\mathrm{r}, \mathrm{s}, \mathrm{t}>\mathrm{s}
$$

the terminal, $\operatorname{Hom}(Y, Z)\left(\delta^{0}\right)$, by

$$
\langle r, s, t>\mapsto t
$$

EXAMPLE. If $Y$ is discrete, 1.e. $Y[1]=\emptyset$, then $\operatorname{Hom}(Y, Z)$ has all functions $\mathrm{Y}[0] \rightarrow Z[0]$ as vertices and has a unique edge joining each pair of vertices.

In general, the set of edges joining points $s, t \in \operatorname{Hom}(Y, Z)[0]$ is given by the set of functions $\mathrm{r}: \mathrm{Y}[1] \rightarrow Z[1]$ satisfying (4.2). Although this construction satisfies the adjoint property $(4.1)$, it seems to have little relation to graph homorphisms.

Next, consider $\left[\underline{\underline{B}}^{\mathrm{OP}}\right.$, Ens]. From Figure 7, it is clear that $\underline{\underline{B}}(,,[0])$ has one vertex and one edge; so that 


$$
\underline{B}(,[0]) \times Y \simeq Y \text {. }
$$

Thus, $\operatorname{Hom}(Y, Z)[0]$ is the set of $\underline{\underline{B}-g r a p h}$ morph1sms:

$$
\begin{aligned}
\operatorname{Hom}(\mathrm{Y}, \mathrm{Z})[0] & \equiv\left[\underline{\underline{B}}^{\mathrm{OP}}, E n s\right](\underline{\underline{B}}(,,[0]) \times \mathrm{Y}, \mathrm{Z}) \\
& \cong\left[\underline{\underline{B}}^{\mathrm{OP}}, \text { Ens }\right](\mathrm{Y}, Z) .
\end{aligned}
$$

Each "edge" of $\operatorname{Hom}(Y, Z)[1]$ is represented by a triple $\langle\mathrm{r}: \mathrm{Y}[1] \rightarrow Z[1] ; \mathrm{s}: \mathrm{Y} \rightarrow \mathrm{Z} ; \mathrm{t}: \mathrm{Y} \rightarrow \mathrm{Z}\rangle$, where $s$ and $t$ are $\underline{\underline{B}-g r a p h}$ morphisms for which $(4.3)$ is commutative:

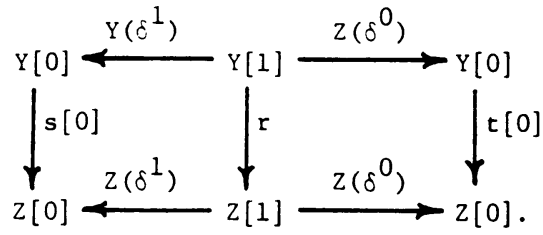

As before,

$$
<r, s, t>\mapsto s
$$

gives the origin of $\operatorname{Hom}(Y, Z)$ and

$$
<r, s, t>r
$$

defines the terminal.

If $s$ and $t$ are given, then the set of edges of $\operatorname{Hom}(Y, Z)$ with origin $s$ and terminal $t$ is indexed by those functions $r: Y[1] \rightarrow Z[1]$ satisfying (4.3). The definition of Hom graph in Ribenboim [5], p. 165, was in this spirit. However, for no apparent reason, only the restriction of $r$ to $Y\left(\sigma^{0}\right) Y[0]$ entered into that definition. In Ribenboim [6], p. 110, a totally different definition was given. Again, it was an explicit construction, and again there was no claim of naturality. The idea was that the edges should be indexed by the functions $r: Y[1] \rightarrow Z[1]$ of our definition. The difficulty is that (4.3) determines only $s[0]$ and $t[0]$, so that the vertices could only be the equivalence classes of "graph homomorphisms restricting to the same functions on vertices". By contrast, our construction is no more cumbersome than these two attempts, but the underly general principle is quite simple and guarantees the Hom will have the proper adjoint relation to cartesian product.

Our internal Hom-functor has the usual properties of Hom in Ens in particular, there is a composition

$$
0: \operatorname{Hom}(Y, Z) \times \operatorname{Hom}(W, Y) \rightarrow \operatorname{Hom}(W, Z) .
$$

The realizations of vertices in $\operatorname{Hom}(Y, Z)[0]$ as graph homomorphisms and of $\operatorname{Hom}(Y, Z)[1]$ as triples $\langle r, s, t\rangle$ are compatible with the composition (4.4).

The composition (4.4) induces a natural monold structure on Hom(W,W). If the invertible elements of $\operatorname{Hom}(\mathrm{W}, \mathrm{W})$ are selected, the resulting subgraph is a group called Aut (W).

The various reflective subcategories constructed in Section 2 and Section 3 in the discussion of categories of graphs sould be cartesian closed. In fact, the following proposition gives an easy computation of Hom in many cases. 
PROPOSITION. Suppose $\Gamma$ is a cartesian closed category and $I: \Gamma^{\prime} \rightarrow \Gamma$ is a full reflective inclusion with $R: \Gamma \rightarrow \Gamma^{\prime}$ the reflector (i.e., $\langle R, I\rangle$ adjoint pair). Then $R$ preserves products iff for all $A \in \Gamma$ and $B \in \Gamma^{\prime}, \operatorname{Hom}(A, B)$ is in $\Gamma^{\prime}$.

PROOF. See Freyd [9; p. 13].

REMARK. The reflective subcategory $A_{1}$ of $\left[\stackrel{A}{=}^{O P}, E n s\right]$ constructed in Section 2 does not satisfy this property; however $A_{2}$ does. ACKNOWLEDGEMENT. We give special thanks to F. E. J. Linton for his advice during the writing of this paper. Thanks are also due to M. L. Gardner, P. Hell and S. MacLane for their encouragement and useful conversations.

\section{REFERENCES}

1. HELL, P. An Introduction to the Category of Graphs (in Topics in Graph Theory, New York, 1977) Ann. New York Acad. Sc1. 328 (1979), 120-136.

2. MACLANE, S. Categories for the Working Mathematiclan, Springer-Verlag, New York, 1971.

3. HARARY, F. and READ, R.C. Is the Null Graph a Pointless Concept?, Graph Theory and Combinatorics, Springer-Verlag, 1973, 37-44.

4. FARZAN, M. and WALLER, D.A. Kronecker Products and Local Joins of Graphs, Canad. J. Math. 29 (1977), 255-269, MR 55\#2625.

5. RIBENBOIM, P. Graphs with Algebraic Structures, Report to the Algebra Group, Queens Papers in Pure and Applied Math. 36, Queens University (1973), 155-184.

6. RIBENBOIM, P. Algebraic Structures on Graphs, Algebra Universal1s 16 (1983), 105-123.

7. MACDONALD, J. and STONE, A. Topo1 Over Graphs, Cahiers Topologie Geom. Differentiable 25 (1984), 51-62.

8. LAMBEK, J. and RATTRAY, B.A. Localization and Duality in Additive Categories, Houston J. Math. I $(1975), 87-100$.

9. FREYD, P. Aspects of Topo1, Bull. Austral. Math. Soc. $\underline{7}$ (1972), 1-76. 


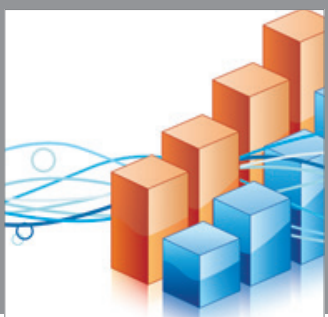

Advances in

Operations Research

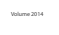

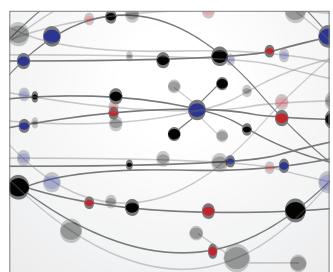

\section{The Scientific} World Journal
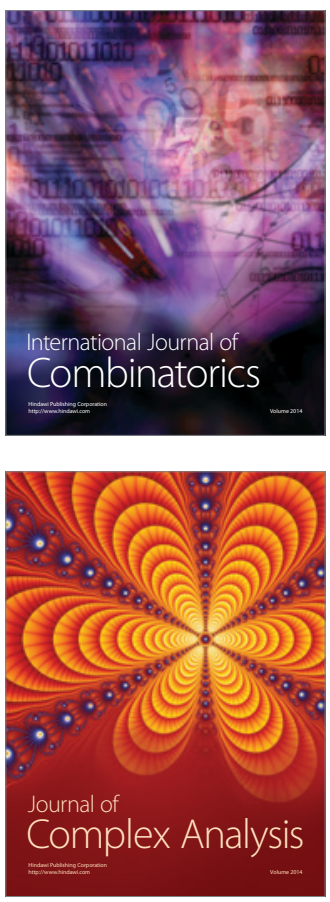

International Journal of

Mathematics and

Mathematical

Sciences
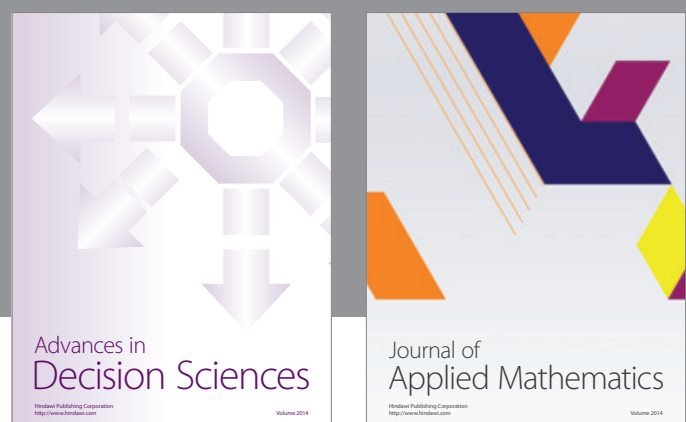

Journal of

Applied Mathematics
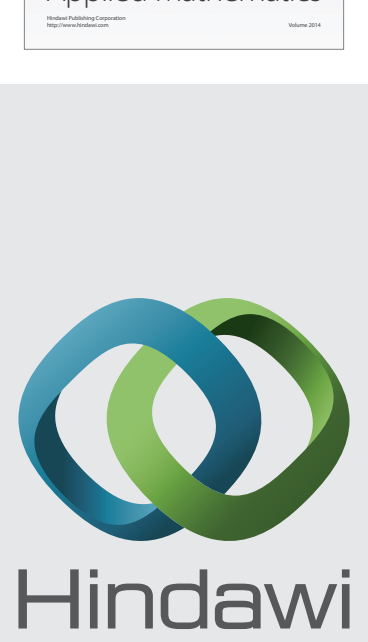

Submit your manuscripts at http://www.hindawi.com
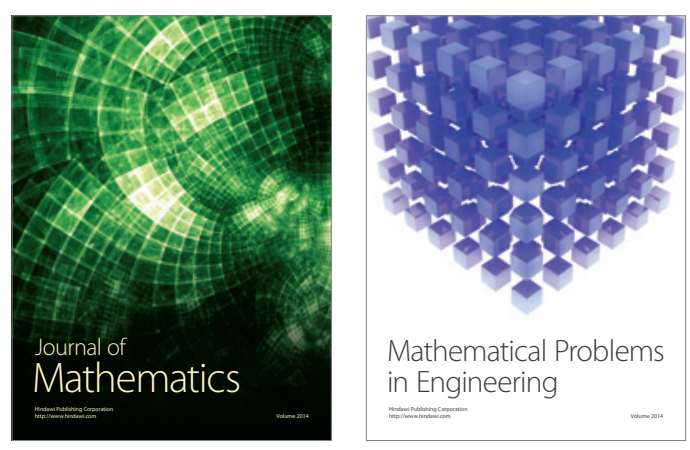

Mathematical Problems in Engineering
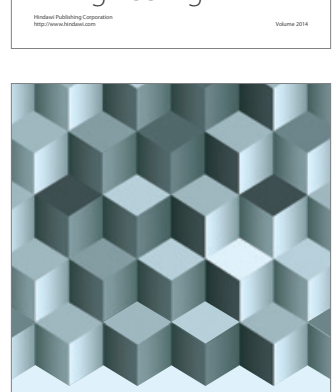

Journal of

Function Spaces
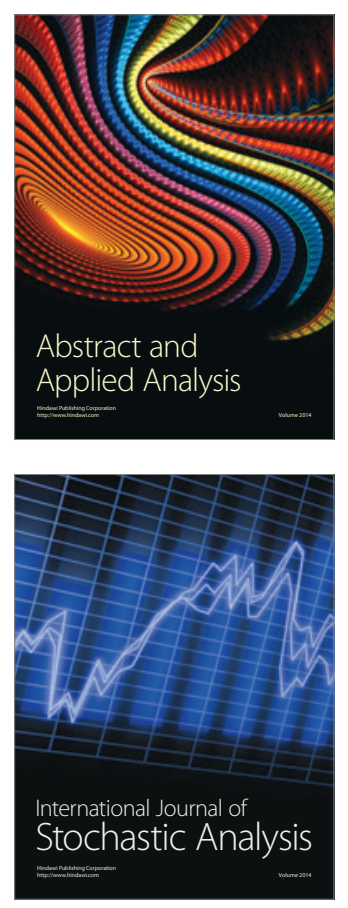

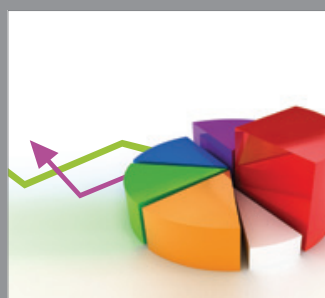

ournal of

Probability and Statistics

Promensencen
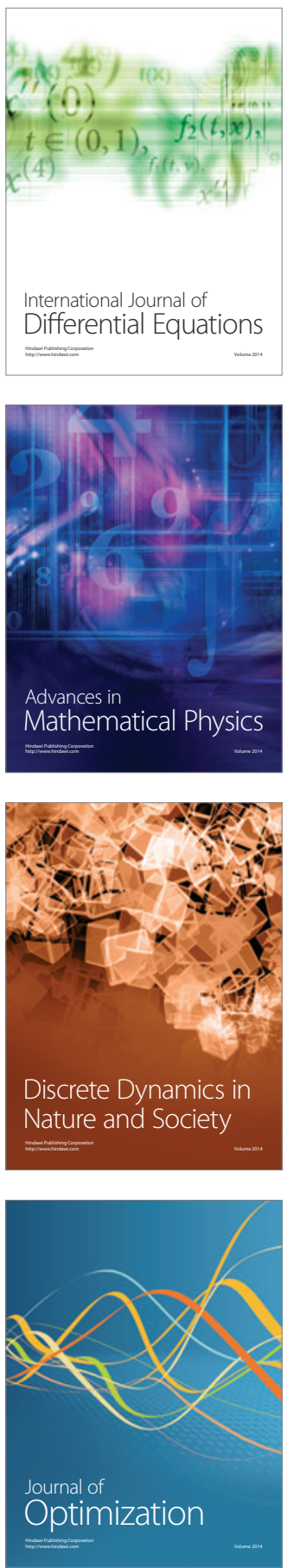